\title{
Cholecystitis/cholangitis secondary to drainage of the biliary tree into an obstructed duodenum: a new technique with a novel complication?
}

A previously well, 73-year-old woman presented with chest pain and was noted to have markedly abnormal cholestatic liver function tests. Magnetic resonance cholangiopancreatography (MRCP) demonstrated a multicystic mass in the pancreatic head with possible extrinsic biliary compression. She underwent endoscopic ultrasound (EUS) with cytological aspiration, which demonstrated dysplastic cellular material.

Multidisciplinary review of the imaging and pathology concluded that she had locally advanced pancreatic cancer involving the hepatic artery. An endoscopic retrograde cholangiopancreatography (ERCP) failed to reach the second part of the duodenum because of tumor ingrowth. She then underwent EUS-guided biliary drainage and a 4-cm fully covered metal stent was inserted ( $>$ Video 1 ).

After this procedure, her jaundice improved and plateaued. She developed epigastric pain that failed to settle. Computed tomography (CT) scans showed a thick-walled gall bladder suggestive of acute cholecystitis and a grossly distended stomach that suggested duodenal obstruction ( $\triangleright$ Fig. 1; $>$ Video 2 ). She proceeded to a laparoscopic cholecystectomy, during which the gallbladder was noted to be edematous. Furthermore, the cholangiogram demonstrated a patent cystic duct with free flow of contrast though the stent into the first part of the duodenum but absent flow distally ( $>$ Fig.2). On-table gastroscopy confirmed that she had complete duodenal obstruction. A self-expanding metal stent (SEMS) was placed across the stricture ( $\triangleright$ Fig.3). The patient subsequently recovered well and was discharged home.

EUS-guided biliary drainage is an alternative to percutaneous access in circumstances where ERCP has failed. Indications include unsuccessful biliary cannulation or an inaccessible papilla [1]. Two major approaches have been described,
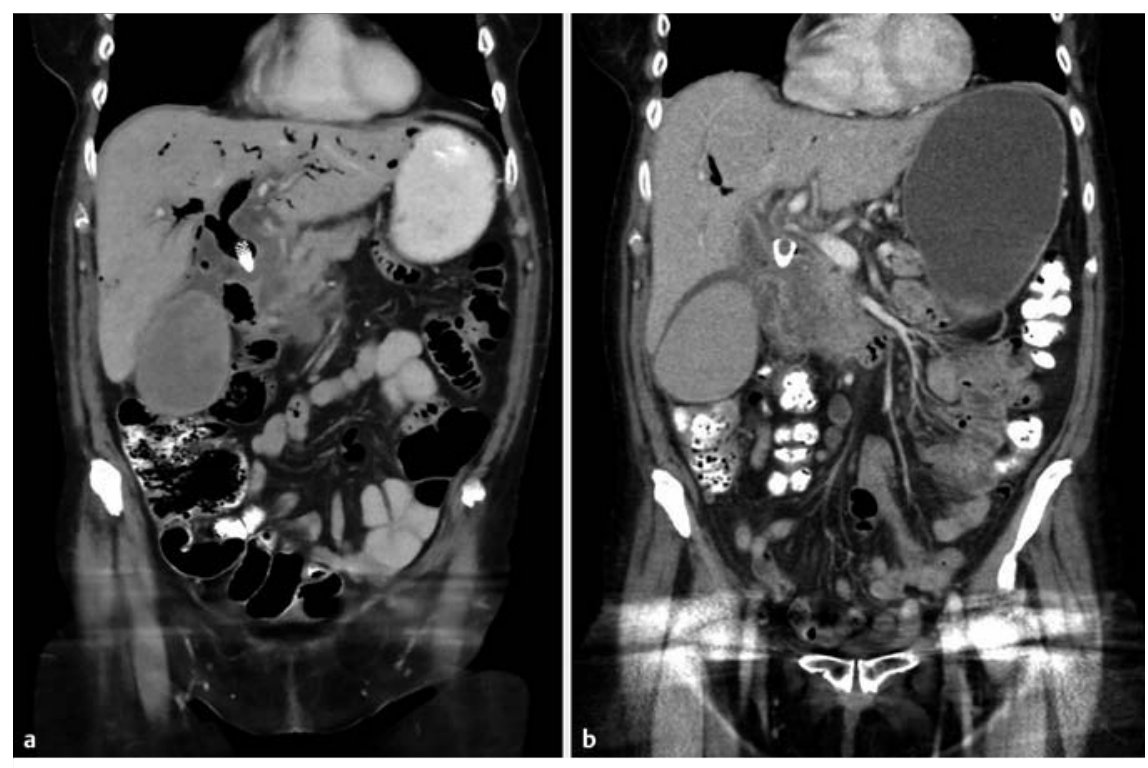

- Fig. 1 Serial computed tomography (CT) scans showing the development of cholecystitis and the progression of gastric dilatation.

either the transgastric or transduodenal routes [2]. Concomitant duodenal and biliary obstruction is not uncommon in advanced pancreatic cancer and this can further complicate biliary access and drainage. We postulate that, following the EUS-guided choledochoduodenostomy, our patient developed biliary sepsis due to progression of the duodenal obstruction distal to the choledochoenteric stented anastomosis. This caused contamination of the biliary tree with gastric contents and subsequent cholangitis.

Endoscopy_UCTN_Code_CPL_1AK_2AI

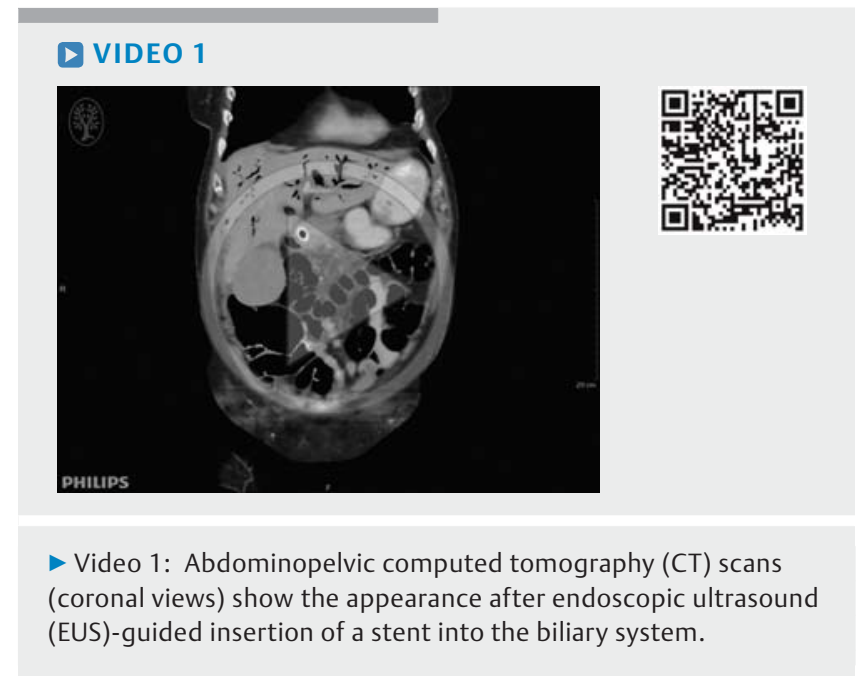




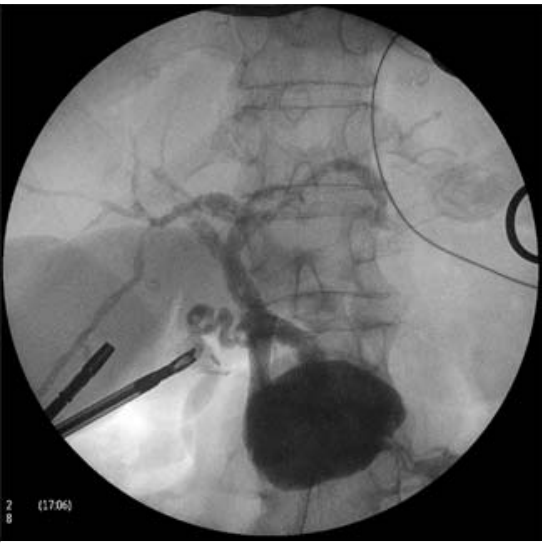

- Fig. 2 Cholangiogram showing free flow of contrast through the stent into the upper part of the duodenum but failure of passage of contrast to D2.

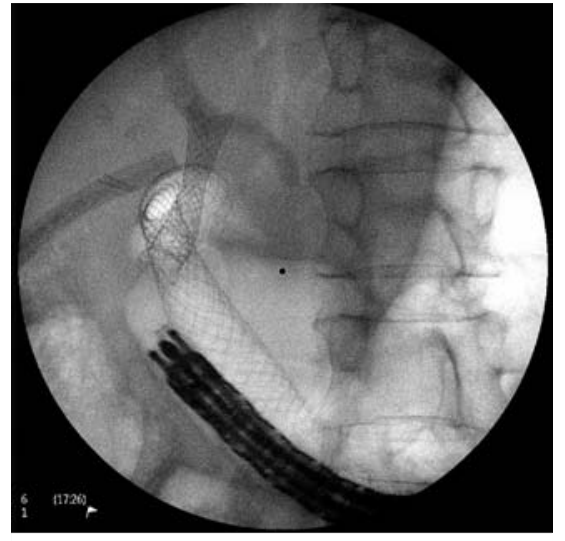

Fig. 3 Radiographic image showing the placement of a duodenal stent. Note: residual contrast is still present in the bile duct.

Competing interests

None

The Authors

Travis Ackermann, Simon Hew, Paul Cashin, Michael Swan, Daniel Croagh

Upper Gastrointestinal Surgery Department, Monash Medical Centre, Clayton, Victoria, Australia

\section{Corresponding author}

\section{Travis Ackermann, MB, BS}

Upper Gastrointestinal Surgery Department, Monash Medical Centre Clayton, 242-246

Clayton Road, Clayton, Victoria 3168.

Australia

travisackermann@bigpond.com
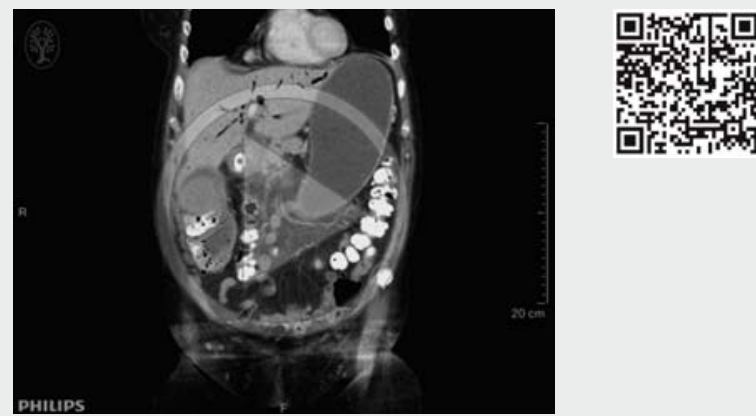

\section{References}

[1] Kim TH, Lim SH, Oh H] et al. Endoscopic ultrasound-guided biliary drainage with placement of a fully covered metal stent for malignant biliary obstruction. World J Gastroenterol 2012; 18: 2526-2532

[2] Tyberg A, Desai AP, Kumta NA et al. EUSguided biliary drainage after failed ERCP: a novel algorithm individualized based on patient anatomy. Gastrointest Endosc 2016; 84: $941-946$

Video 2: Abdominopelvic computed tomography (CT) scans (coronal views) show the gastric outlet obstruction after insertion of the stent. 